\section{A) Check for updates}

Cite this: Food Funct., 2019, 10, 4440

\title{
The key role of ovalbumin in lipid bioaccessibility and oxidation product profile during the in vitro digestion of slightly oxidized soybean oil $\dagger$
}

\begin{abstract}
A. S. Martin-Rubio, P. Sopelana and M. D. Guillén (D)*
The behaviour of slightly oxidized virgin and refined soybean oils during in vitro digestion was studied by ${ }^{1} \mathrm{H}$ nuclear magnetic resonance $\left({ }^{1} \mathrm{H}\right.$ NMR) and solid phase microextraction-gas chromatography/mass spectrometry. The main objectives were to analyze lipolysis extent and oxidation during digestion, and to assess the impact of two different proportions of ovalbumin on both processes. At the same time $\gamma$-tocopherol fate was monitored, when possible, by ${ }^{1} \mathrm{H}$ NMR. The results reveal that the initial oxidation degree of the oils negatively influences the lipolysis extent, reducing the bioaccessibility of the major oil components, which include some essential fatty acids. Although the low ovalbumin proportion tested does not significantly affect lipolysis, this is greatly enhanced when ovalbumin is added at a high level, improving lipid bioaccessibility. It has also been shown that oxidation does not seem to have occurred to a great enough extent during digestion for it to be detected from polyunsaturated acyl group degradation. Moreover, the changes observed in the oxidation product profile of the starting oils after digestion can be considered to be due mainly to the transformation of the initially present hydroperoxides, whose concentration diminishes in the digested samples to give hydroxy-dienes, epoxides and aldehydes. In presence of a high ovalbumin proportion, hydroperoxide reduction to hydroxy-dienes is favoured and lower levels of aldehydes and epoxides are observed. This latter could be due to a diminution in their generation and/ or to their reaction with ovalbumin. A high proportion of this protein in the system also increases $\gamma$-tocopherol bioaccessibility.
\end{abstract}

Received 21st March 2019 Accepted 25th June 2019 DOI: 10.1039/c9fo00598f rsc.li/food-function and safety of food can be adversely affected, since not only losses of essential lipophilic nutrients like polyunsaturated fatty acids or certain antioxidants can occur, ${ }^{3,6,9}$ but also compounds with negative biological implications, such as oxygenated $\alpha, \beta$-unsaturated aldehydes, ${ }^{10}$ could be generated. ${ }^{5}$ In this context, the unsaturation degree of lipids and their oxidation extent have been found to affect the oxidation progress throughout digestion. . $^{3,6,7,9}$

Another element that can influence the evolution of digested lipids in the gastrointestinal tract is the presence of other nutrients, among them proteins. In this sense, NievaEchevarría, Goicoechea and Guillén ${ }^{4}$ investigated the effect of two proteins (ovalbumin and soy protein isolate) on the in vitro digestion process of slightly oxidized sunflower and flaxseed oils, using quite a high proportion of proteins to lipids, in order to simulate the ratio that might exist between these two types of nutrients in a model food system. It was observed that the presence of protein during the in vitro digestion of edible oils affects the extent of lipolysis and of oxidation reactions taking place during this process; this can be explained both by the emulsifying properties of this type of compounds ${ }^{11}$ and by the antioxidant ability of proteins and peptides, ${ }^{12}$ which might 
favour and limit, respectively, both types of reactions. However, the impact of proteins on oxidation reactions also depends on their composition, since iron-containing proteins (heme proteins) could promote oxidation during digestion. ${ }^{13}$

Bearing in mind all the above, and with the aim of contributing towards building a solid body of knowledge concerning the complex process of lipid digestion, which is directly relevant to the health effects of dietary lipids, the study under in vitro digestion conditions of slightly oxidized virgin and refined soybean oils was tackled in this work. These oils were selected as examples of linoleic-rich vegetable oils but, unlike the sunflower oil previously studied, ${ }^{6}$ with a certain proportion of linolenic acyl groups and a different profile in minor components when compared with the latter. ${ }^{14}$ The main difference between these two oils in their unoxidized state laid in their composition in minor components. ${ }^{15}$ In this context, the fate during in vitro digestion of $\gamma$-tocopherol $(\gamma$-T), the main tocopherol present in soybean oil, ${ }^{14}$ was monitored when possible. The use of slightly oxidized oils will show the extent to which their initial lipid degradation status can affect the progress of lipolysis and oxidation during in vitro digestion. In addition, the influence on these processes of the presence of two fairly different proportions of ovalbumin, a non-heme protein widely employed as an ingredient in many food formulations, was also addressed.

The techniques employed were proton nuclear magnetic resonance $\left({ }^{1} \mathrm{H}\right.$ NMR $)$ and solid phase microextraction followed by gas chromatography/mass spectrometry (SPME-GC/MS). ${ }^{1} \mathrm{H}$ NMR allows one to study simultaneously the lipolysis degree, the oxidation extent and the evolution of $\gamma$-T through the in vitro digestion of the selected oils. This technique is able to provide information about oxidation products that can be either bound or unbound to acyl groups. SPME-GC/MS, in turn, constitutes a complementary and very useful tool that, thanks to its higher sensitivity and specificity, makes it possible to obtain further information in relation to the occurrence of oxidation and even to confirm the results obtained by using ${ }^{1} \mathrm{H}$ NMR.

\section{Materials and methods}

\subsection{Samples subjected to in vitro digestion}

The samples subjected to in vitro digestion were two slightly oxidized commercial soybean oils: one virgin (VSx) and the other refined (RSx). They were obtained after submitting the corresponding fresh oils to accelerated storage conditions, as in previous works. ${ }^{16,17}$ To this aim, $10 \mathrm{~g}$ of each of the fresh oils were weighed in glass Petri dishes of $80 \mathrm{~mm}$ diameter and placed in a convection oven (Memmert $\mathrm{GmbH}+\mathrm{Co}$, Schwabach, Germany) at $70{ }^{\circ} \mathrm{C}$ with circulating air for 4 and 5 days in the case of the virgin and the refined oils respectively, in order to obtain oils with a similar oxidation degree.

In addition, samples were prepared by mixing each of these two slightly oxidized oils with two different proportions of ovalbumin; $0.26 \mathrm{~g}$ of ovalbumin per $\mathrm{g}$ of oil in the samples with the low level of ovalbumin (VSx + LO and RSx + LO) and $2.6 \mathrm{~g}$ of ovalbumin per $\mathrm{g}$ of oil in the ones with the high ovalbumin proportion ( $\mathrm{VSx}+\mathrm{HO}$ and $\mathrm{RSx}+\mathrm{HO}$ ), this latter corresponding to a dose previously used in another study. ${ }^{4}$ Food grade ovalbumin was acquired from a protein manufacturer (Apasa SA, Astigarraga, Spain).

\subsection{In vitro digestion}

Samples (0.5 g) of the two oils, both in the absence and in presence of ovalbumin, were digested following the same procedure as in previous works, ${ }^{6,7}$ based on the static in vitro gastrointestinal model developed by Versantvoort, Oomen, Van de Kamp, Rompelberg and Sips; ${ }^{18}$ this was slightly modified in our laboratory in order to reach a higher level of lipolysis. ${ }^{19}$ This in vitro digestion model involves a three-step procedure to simulate digestive processes in the mouth, stomach, and small intestine, by sequentially adding the corresponding digestive juices. More details about the in vitro digestion procedure can be found in the ESI. $\dagger$

All the reagents used were acquired from Sigma-Aldrich (St Louis, MO, USA). Two digestion experiments, each including duplicate samples of the two studied oils, were performed. Blank samples corresponding to the mixture of juices submitted to digestive conditions were also taken for further analysis.

\subsection{Lipid extraction of the digestates}

Lipids of the digestates were extracted using dichloromethane as solvent (HPLC grade, Sigma-Aldrich) and following the same methodology as in previous studies, ${ }^{20}$ which involves a three-stage liquid-liquid extraction process with $20 \mathrm{ml}$ of dichloromethane each. Afterwards, to ensure a complete protonation of fatty acids and/or the dissociation of the potential salts formed, the remaining water phase was acidified to $\mathrm{pH} 2$ with $\mathrm{HCl}(37 \%)$ and a second extraction was carried out, also in three steps. ${ }^{6,7}$ Given that in the case of the samples with a high proportion of ovalbumin a strong emulsion is formed when mixing the digested sample with the extraction solvent, which makes it difficult to separate the aqueous and lipid phases, the extraction was performed with the aid of a centrifuge in order to break up this emulsion. For this purpose, a Sigma $3 \mathrm{~K} 30$ centrifugal machine working at $10000 \mathrm{rpm}$ was used (Sigma Laboratory Centrifuges, Germany), each extraction step lasting $10 \mathrm{~min}$. This same extraction procedure was used for all the samples, without any differences in extraction efficiencies achieved when using either centrifugation or extraction with separating funnels in the case of the samples without ovalbumin and with a low proportion of this protein. All the dichloromethane extracts of each sample were mixed and the solvent was eliminated by means of a rotary evaporator under reduced pressure at room temperature, in order to avoid lipid oxidation. Afterwards, these extracts were stored at $-80^{\circ} \mathrm{C}$ until their analysis.

\subsection{Analysis by ${ }^{1} \mathrm{H}$ NMR}

2.4.1. Operating conditions. The ${ }^{1} \mathrm{H}$ NMR spectra of the starting oils (VSx and RSx), of the lipid extracts of their corres- 
ponding digestates (DVSx and DRSx), and of the extracts obtained from the digestates of the oil samples containing a low proportion of ovalbumin $(\mathrm{D}(\mathrm{VSx}+\mathrm{LO})$ and $\mathrm{D}(\mathrm{RSx}+\mathrm{LO}))$, and a high proportion of this protein $(\mathrm{D}(\mathrm{VSx}+\mathrm{HO})$ and $\mathrm{D}(\mathrm{RSx}$ $+\mathrm{HO})$ ) were acquired in quadruplicate using a Bruker Avance 400 spectrometer operating at $400 \mathrm{MHz}$. For this purpose, the above-mentioned lipid samples (approximately $0.16 \mathrm{~g}$ ) were dissolved in $400 \mu \mathrm{l}$ of deuterated chloroform, which contained tetramethylsilane (TMS) as internal reference (Cortec, Paris, France). The acquisition conditions were the same as those used in previous studies. ${ }^{21,22}$

2.4.2. Identification of some lipid components. The identification of the oil acyl groups, of partial glycerides, of $\gamma$-T and of the oxidation products present in the various samples was carried out on the basis of the signal assignments shown in Table S1 (see ESI $\dagger$ ), made from bibliographic data and with the aid of several standard compounds. These were: $\gamma$-T, acquired from Sigma-Aldrich, and cis-(12,13)-epoxy-9 $(Z), 15(Z)$-octadecadienoic acid, acquired from Cymit Quimica (Barcelona, Spain).

2.4.3. Quantification from ${ }^{1} \mathbf{H}$ NMR spectral data. Bearing in mind that the area of each ${ }^{1} \mathrm{H}$ NMR spectral signal is proportional to the number of protons that generate it, and that the proportionality constant is the same for all kinds of protons, the area of some spectral signals can be employed to quantify: (i) the molar percentages of the different types of glycerides; (ii) the molar concentrations of linolenic and linoleic acyl groups + fatty acids, referring to the total of acyl groups + fatty acids; (iii) the molar concentration of $\gamma$-T, referring to the total of acyl groups + fatty acids; and (iv) the molar concentrations of several oxidation compounds present in the starting oils and/or in the lipid extracts of the digestates, referring to the total of acyl groups + fatty acids, following the procedures indicated below.

Various types of glycerides and glycerol. The molar percentages of triglycerides (TG\%), diglycerides (1,2-DG\% and 1,3DG\%), monoglycerides (2-MG\% and $1-\mathrm{MG} \%$ ) and glycerol in relation to the total number of glyceryl structures present in the lipid samples were determined using the equations developed and validated in previous studies. ${ }^{20,23}$ All these equations are given as ESI (see eqn (S1)-(S10)†).

Another parameter relating to lipolysis extent can also be determined from the data relative to the different types of glycerides, namely lipid bioaccessibility $\left(\mathrm{L}_{\mathrm{BA}}\right)$. This takes into account only the molecules that are directly absorbable, monoglycerides and fatty acids, in relation to the total number of acyl groups + fatty acids present in the sample. This parameter was calculated by using eqn (S11) and (S12). $\dagger$

Lipid composition. The concentrations of linolenic and linoleic acyl groups + fatty acids were estimated in millimoles per mole of the sum of acyl groups + fatty acids $\left(\mathrm{mmol} \mathrm{mol}^{-1} \mathrm{AG}+\right.$ FA), both in the starting oils and in the lipid extracts of the digested samples, by using eqn (S13) and (S14). $\dagger$

Oxidation compounds. The molar concentrations of the different types of oxidation products present in the starting oils and in the lipid extracts of the digested samples, referring to the total of acyl groups + fatty acids and expressed as mmol $\mathrm{mol}^{-1} \mathrm{AG}+\mathrm{FA}$, were also estimated as in a previous study ${ }^{4}$ by using eqn (S15). $\dagger$

It must be noticed that, as shown in Fig. $\mathrm{S} 1$ for $\mathrm{D}(\mathrm{VSx}+\mathrm{HO})$ (see ESI $\dagger$ ), in the case of the samples digested with a high ovalbumin proportion, signals coming from the protein used are perceived in the ${ }^{1} \mathrm{H}$ NMR spectra of their corresponding digestates. Some of these overlap with the signals used to estimate both the concentration of epoxides giving signal at $2.9 \mathrm{ppm}$ (see signals "e1-e4" in Table S1 $\dagger$ ) and the molar percentages of 1,3-DG and TG (see signals "M" and "O", respectively), so their contribution must be subtracted, especially in the case of epoxides. For this purpose, ovalbumin was added to the digestive juices after undergoing the digestion process and this mixture was extracted in the same way as the rest of digested samples; the relative areas of the different ovalbumin signals can be determined from the corresponding ${ }^{1} \mathrm{H}$ NMR spectrum, free of lipids. This enables one to subtract the area of the signals overlapping with those of lipid components in the spectra of the extracts obtained from the digested oil samples taking as a reference the signals that do not overlap with one another (see Fig. S1 $\dagger$ ). It is worth noticing that while some signals coming from ovalbumin also overlap with those of bis-allylic protons (signals " $\mathrm{H}+\mathrm{G}$ "), their area is very small in relation to that of the latter, and so can be ignored.

$\gamma-T$. The concentration of $\gamma$-T was estimated from signal "h" (see Table S1†), in the same way as described for oxidation compounds (see eqn (S16)†).

\subsection{Study of oxidation during in vitro digestion by SPME-GC/MS}

2.5.1. SPME analysis. The extraction of the volatile components of the several digestates $(0.5 \mathrm{~g}$ in $10 \mathrm{ml}$ screw-cap vials) was carried out automatically by using a CombiPAL autosampler (Agilent Technologies, Santa Clara, CA, USA), in the same way as in previous works. ${ }^{4,6,7}$

Given that the nature of the samples subjected to the digestion process (oil samples) is very different from that of the digestates (with a basically aqueous matrix), it is necessary to prepare mixtures of the non-digested oils with the digestive juices after being submitted to the digestion process, in the same proportions as in the digestates; this enables one to make an adequate assessment by SPME-GC/MS of the changes taking place throughout the in vitro digestion process. Therefore, the samples subject of study, which were analyzed in duplicate, were the following: (i) the digestates both of the slightly oxidized soybean oil samples (DVSx and DRSx), and of the slightly oxidized oil samples plus ovalbumin at the two levels of concentration ( $(\mathrm{VSx}+\mathrm{LO}), \mathrm{D}(\mathrm{RSx}+\mathrm{LO})$, $\mathrm{D}(\mathrm{VSx}+\mathrm{HO})$ and $\mathrm{D}(\mathrm{RSx}+\mathrm{HO}))$; (ii) the digestive juices after being submitted to digestion conditions (DJ); and (iii) the mixtures made up of the starting oils and DJ (VSx + DJ and $\mathrm{RSx}+\mathrm{DJ})$.

The fiber used, coated with divinylbenzene/carboxen/ polydimethylsiloxane (DVB/CAR/PDMS, 50/30 $\mu \mathrm{m}$ film thick- 
ness, $1 \mathrm{~cm}$ long), was acquired from Supelco (Sigma-Aldrich); this was inserted into the headspace of the sample and was maintained for $55 \mathrm{~min}$ at $50{ }^{\circ} \mathrm{C}$, after a pre-equilibration time of $5 \mathrm{~min}$.

\subsubsection{GC/MS study}

Operating conditions. The fiber containing the extracted components was desorbed for $10 \mathrm{~min}$ in the injection port (splitless mode with 5 min purge time) of a 7890A gas chromatograph equipped with a 5975C inert MSD with Triple Axis Detector (Agilent Technologies) and a computer operating with the ChemStation program. The column used was a fused-silica capillary column $(60 \mathrm{~m}$ long $\times 0.25 \mathrm{~mm}$ inner diameter $\times$ $0.25 \mu \mathrm{m}$ film thickness, from Agilent J\&W Advanced Capillary GC Columns), coated with a non-polar stationary phase (HP-5MS, 5\% phenyl methyl siloxane). The operating conditions were as follows: the oven temperature was set initially at $50{ }^{\circ} \mathrm{C}$ ( 5 min hold) and increased to $290{ }^{\circ} \mathrm{C}$ at $4{ }^{\circ} \mathrm{C} \mathrm{min}^{-1}$ ( 2 min hold); the temperatures of the ion source and of the quadrupole mass analyser were kept at $230{ }^{\circ} \mathrm{C}$ and $150{ }^{\circ} \mathrm{C}$ respectively; helium was used as carrier gas at a pressure of 18.611 psi; injector temperature was held at $250{ }^{\circ} \mathrm{C}$; mass spectra were recorded at an ionization energy of $70 \mathrm{eV}$, and the data acquisition mode employed was scan. In order to avoid carry-over problems between samples, after each run the fiber was submitted to heating at $250{ }^{\circ} \mathrm{C}$ for $20 \mathrm{~min}$ in the Fiber Cleaning and Conditioning Station of the CombiPAL autosampler.

A reference sample of known composition was periodically analyzed in order to verify not only the extraction efficiency and repeatability of the SPME fiber but also the performance of the equipment.

Identification of the compounds present in the headspace of the samples. Most of the components were identified by using commercial standards, acquired from Sigma-Aldrich. These were: pentanal (base peak: 86), hexanal (100), heptanal (114), octanal (128), nonanal (142), (E)-2-pentenal (84), $(E)$-2-hexenal (98), (E)-2-heptenal (112), (E)-2-octenal (126), (E)-2-nonenal (140), $(Z, E)$-2,4-heptadienal (110), (E,E)-2,4-heptadienal (110), $(Z, E)$-2,4-nonadienal (138), (E,E)-2,4-nonadienal (138), $(Z, E)-2,4$ decadienal (152), (E,E)-2,4-decadienal (152) and 2-pentyl-furan (138).

When standards were not available, matching of the mass spectra with those obtained from scientific literature or from a commercial library at higher than 85\% (Wiley W9N08, Mass Spectral Database of the National Institute of Standards and Technology (NIST)), was taken as identification criterion.

Semi-quantification of the compounds present in the headspace of the samples. This was based on the area counts of the base peak (Bp) of the mass spectrum of each compound divided by $10^{6}$. When the Bp of a compound overlapped with the same ion peak of the mass spectrum of another compound, an alternative ion peak was selected for the semi-quantification of the former. The area counts thus determined are useful for the comparison of the abundance of each compound in the different samples.

\subsection{Statistical analysis}

The significance of the differences between the various determinations made among the samples was determined by oneway variance analysis (ANOVA) followed by Tukey b test at $p<0.05$, using SPSS Statistics 24 software (IBM, NY, USA).

\section{Results and discussion}

\subsection{Characterization of the starting oil samples}

As mentioned in section 2.1, the samples selected for the study were two slightly oxidized soybean oils: one virgin and the other refined. The concentrations of their polyunsaturated acyl groups (linolenic and linoleic), and of some oxidation compounds, all of them determined by ${ }^{1} \mathrm{H}$ NMR and expressed in mmol mol ${ }^{-1} \mathrm{AG}+\mathrm{FA}$, are given in Tables 1 and 2, respectively. Table 2 reveals that both oils have a low oxidation level, their concentration of hydroperoxides (signals "c" and "b" in Fig. 1) being very low, with values of $11.16 \pm 0.22 \mathrm{mmol} \mathrm{mol}^{-1}$ $\mathrm{AG}+\mathrm{FA}$ in the virgin oil and $11.97 \pm 1.21 \mathrm{mmol} \mathrm{mol}^{-1} \mathrm{AG}+\mathrm{FA}$ in the refined one. Both samples also have small concentrations of epoxides (see signal "e" in Fig. 1) and aldehydes are not detected in the ${ }^{1} \mathrm{H}$ NMR spectra of any of these oils. A small amount of hydroxy $(Z, E)$-conjugated dienes can also be noticed in the virgin oil (signal "a" in Fig. 1). The concentrations of tocopherols and squalene are higher in the oxidized refined oil than in the oxidized virgin oil, and the opposite is true for free fatty acids (see Table S2 in the ESI $\dagger$ ).

\subsection{Extent of lipolysis through the in vitro digestion}

3.2.1. Lipolysis in the samples digested without ovalbumin. As one would expect, the main glyceride structures present in the non-digested oils were TG, together with a much lower proportion of 1,2-DG. This can be observed in Table 3, which shows the molar percentages of the several kinds of glycerides and of glycerol, in all the studied samples before and after digestion, in relation to the total number of

Table 1 Concentrations of polyunsaturated AG + FA, expressed as $\mathrm{mmol} \mathrm{mol} \mathrm{m}^{-1} \mathrm{AG}+\mathrm{FA}$, in the slightly oxidized virgin and refined soybean oil samples, before (VSx and RSx) and after in vitro digestion in the absence of ovalbumin (DVSx and DRSx), with a low proportion of ovalbumin added ( $(V S x+L O)$ and $D(R S x+L O))$ and with a high proportion of ovalbumin $(D(V S x+H O)$ and $D(R S x+H O)$ ). Different letters within each column indicate a significant difference among the samples corresponding to the same type of oil (virgin or refined) $(p<0.05)$ AG + FA: acyl groups + fatty acids

\begin{tabular}{lll}
\hline & Linolenic & Linoleic \\
\hline VSx & $51.4 \pm 1.7 \mathrm{a}$ & $431.3 \pm 2.9 \mathrm{a}$ \\
DVSx & $49.8 \pm 3.2 \mathrm{a}$ & $424.9 \pm 12.5 \mathrm{a}$ \\
D(VSx + LO) & $50.0 \pm 1.0 \mathrm{a}$ & $422.7 \pm 8.4 \mathrm{a}$ \\
D(VSx + HO) & $50.7 \pm 2.6 \mathrm{a}$ & $426.1 \pm 3.6 \mathrm{a}$ \\
& & \\
RSx & $44.3 \pm 1.8 \mathrm{a}$ & $459.5 \pm 12.7 \mathrm{a}$ \\
DRSx & $42.5 \pm 3.0 \mathrm{a}$ & $451.4 \pm 13.3 \mathrm{a}$ \\
D(RSx + LO) & $42.7 \pm 2.8 \mathrm{a}$ & $452.3 \pm 0.7 \mathrm{a}$ \\
D(RSx + HO) & $43.8 \pm 2.1 \mathrm{a}$ & $454.2 \pm 2.2 \mathrm{a}$
\end{tabular}


Table 2 Concentrations of the several kinds of oxidation compounds, expressed in mmol mol $\mathrm{m}^{-1}$ of acyl groups + fatty acids, present in the virgin and refined slightly oxidized soybean oil samples, before (VSx and RSx) and after in vitro digestion in the absence of ovalbumin (DVSx and DRSx), with a low proportion of ovalbumin added $(D(V S x+L O)$ and $D(R S x+L O))$ and with a high proportion of ovalbumin $(D(V S x+H O)$ and $D(R S x+H O))$. Different letters within each column indicate a significant difference among the samples corresponding to the same type of oil (virgin or refined) $(p<0.05)$. CD-OOH: conjugated hydroperoxy-dienes; $\mathrm{CD}-\mathrm{OH}$ : conjugated hydroxy-dienes

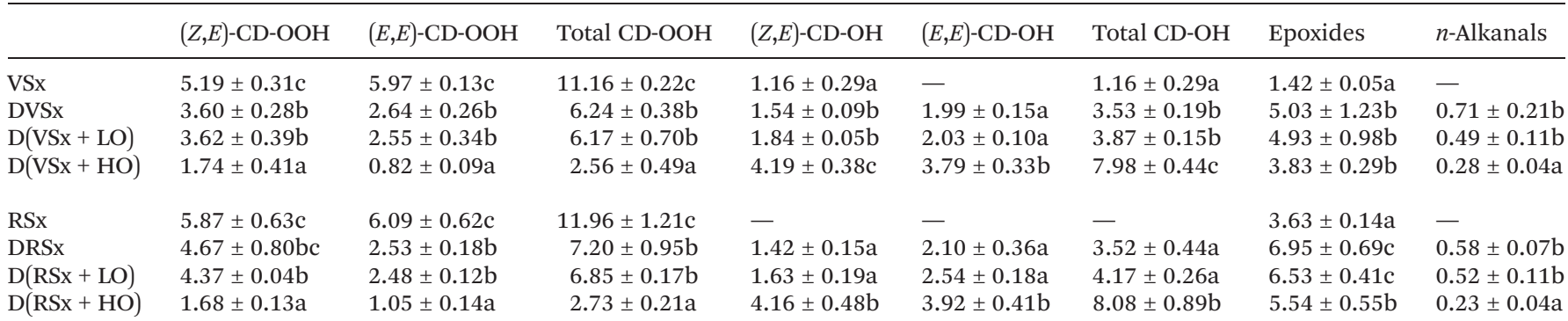

-: not detected.

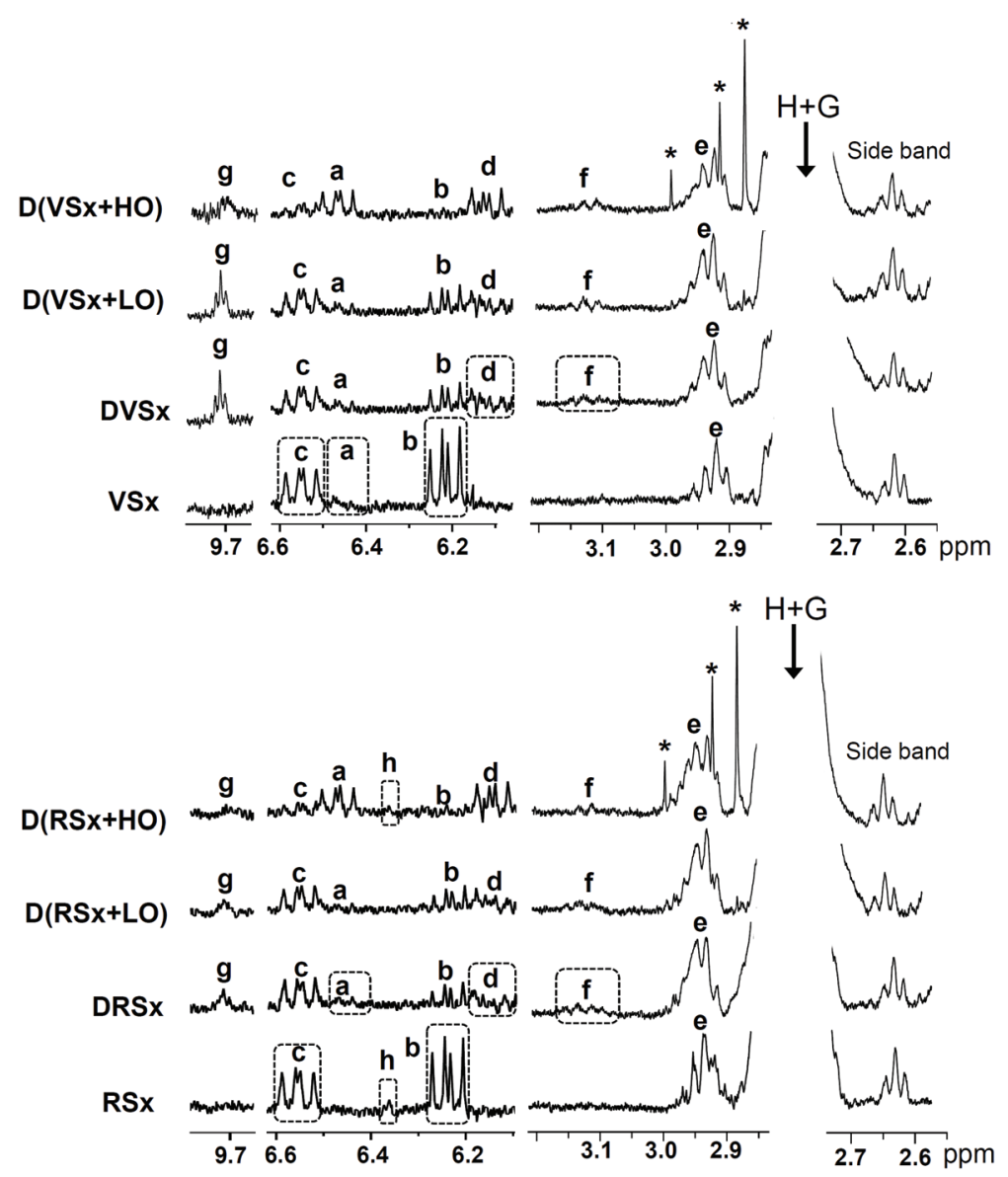

Fig. 1 Enlargements of some regions of the ${ }^{1} \mathrm{H}$ NMR spectra of the slightly oxidized virgin and refined oils (VSx and RSx), and of the lipid extracts obtained after their in vitro digestion process in the absence of ovalbumin (DVSx and DRSx), as well as in the presence of a low (D(VSx + LO) and $D(R S x+L O))$ and a high proportion of ovalbumin $(D(V S x+H O)$ and $D(R S x+H O))$. The signal letters agree with those in Table $S 1, \dagger$ considering that "e" includes signals "e1-e4" and " $f$ " signals " $f 1+f 2$ ". Signals marked with an asterisk are considered to come from the ovalbumin sample used. The plots corresponding to the same ${ }^{1} \mathrm{H}$ NMR spectral region are presented at a fixed value of absolute intensity, for them to be valid for comparative purposes.

glyceryl structures. As this table reveals, the molar percentage of TG greatly diminishes after digestion, reaching values of 30.47 and $31.38 \%$ in the digested virgin and refined oil samples, respectively. These figures are of a similar order to those obtained in earlier works conducted with slightly oxidized samples of other highly unsaturated oils like sunflower ${ }^{6}$ 
Table 3 Molar percentages of triglycerides (TG\%), diglycerides (1,2-DG\% and 1,3-DG\%), monoglycerides (2-MG\% and 1-MG\%) and glycerol (Gol\%) in relation to the total number of glyceryl structures present in the lipid samples, together with lipid bioaccessibility ( $\left.L_{B A}\right)$ parameter, in the slightly oxidized virgin and refined soybean oil samples, before (VSx and RSx) and after in vitro digestion in the absence of ovalbumin (DVSx and DRSx), with a low proportion of ovalbumin added (D(VSx $+L O)$ and $D(R S x+L O))$, and with a high proportion of ovalbumin (D(VSx + HO) and D(RSx + HO)). Different letters within each column indicate a significant difference among the samples corresponding to the same type of oil (virgin or refined) $(p<0.05)$

\begin{tabular}{|c|c|c|c|c|c|c|c|}
\hline DVSx & $30.47 \pm 4.11 \mathrm{~b}$ & $14.15 \pm 2.25 b$ & $3.01 \pm 1.42 b$ & $22.84 \pm 3.53 a$ & $6.41 \pm 0.60 b$ & $23.12 \pm 4.31 \mathrm{a}$ & $58.09 \pm 6.49 a$ \\
\hline $\mathrm{D}(\mathrm{VSx}+\mathrm{HO})$ & $5.26 \pm 0.08 \mathrm{a}$ & $17.86 \pm 0.14 \mathrm{~cd}$ & $1.99 \pm 0.25 \mathrm{a}$ & $39.17 \pm 1.69 b$ & $6.75 \pm 0.87 \mathrm{~b}$ & $28.97 \pm 0.78 a$ & $81.50 \pm 0.01 b$ \\
\hline $\mathrm{RSx}$ & $99.40 \pm 0.08 c$ & $0.62 \pm 0.03 a$ & - & - & - & nd & nd \\
\hline DRSx & $31.38 \pm 4.51 b$ & $14.70 \pm 1.40 \mathrm{bc}$ & $4.26 \pm 1.17 b$ & $22.67 \pm 1.23 \mathrm{a}$ & $6.86 \pm 0.88 b$ & $20.14 \pm 5.20 \mathrm{a}$ & $55.98 \pm 5.94 a$ \\
\hline
\end{tabular}

and flaxseed; ${ }^{7}$ and the same can be said of the relative proportions of the different types of partial glycerides generated during the lipolytic process. Of these, 2-MG and 1,2-DG are the most abundant, while the molar percentages of 1-MG and 1,3DG are much lower.

However, the TG molar percentages in the digested samples are higher than those found when the same unoxidized soybean oils were digested, which were close to $22 \% .^{15}$ The lower hydrolysis extent found in the oxidized oils, which is in agreement with the findings of other authors, ${ }^{24,25}$ could be explained by decreased lipase activity due to the incipient oxidation level of the samples. In fact, it has been reported that hydroperoxides, present in the oxidized oil samples of this work (see section 3.1), could react with amino acid residues of proteins,${ }^{26,27}$ which could lead to a decrease in their functionality in the case of enzymes. Thus, decreased activity of several digestive enzymes, including porcine pancreatic lipase, which is the main lipase used in this study, due to their reaction with lipid hydroperoxides has been reported by some researchers. ${ }^{28-30}$ In addition, other authors ${ }^{31}$ have described that the presence of dimers and polymers generated due to lipid oxidation impairs TG hydrolysis by negatively affecting the activity of pancreatic lipase; however, this type of oxidation products has not been determined in the present work.

The lower lipolysis degree achieved in the digestates of the oxidized oil samples here studied when compared with those of their respective fresh oil samples ${ }^{15}$ is also noticed in the lower molar percentage of glycerol in the former (23.12 and $20.14 \%$ in the virgin and in the refined oil samples, respectively) than in the latter (near 30\%).

3.2.2. Lipolysis in the samples containing ovalbumin. The addition of a low proportion of ovalbumin $(0.26 \mathrm{~g}$ ovalbumin per $\mathrm{g}$ of oil) to the slightly oxidized soybean oil samples does not cause significant variations in the lipolytic process, as can be seen in Table 3. However, in the samples containing a high level of this protein ( $2.6 \mathrm{~g}$ per $\mathrm{g}$ of oil), a very high lipolysis degree is achieved; this can be observed in Table 3 , which shows percentages of remaining TG of $5.26 \%$ and $7.27 \%$ in the digestates of the virgin and the refined oil samples, respectively. The great increment observed in lipolysis could be due to the emulsifying effect of the ovalbumin added, ${ }^{32}$ possibly increased during in vitro digestion due to proteolysis; $;^{33}$ this is expected to improve the dispersion of the oil into the aqueous phase and to reduce the oil droplets size, thus increasing the lipid area exposed to the action of lipases.

It must also be highlighted that the lipolysis extent in the samples digested with a high ovalbumin proportion was considerably higher even than that achieved after the in vitro digestion of the same unoxidized soybean oils..$^{15}$ Therefore, in the light of these findings, one could conclude that the presence of this high level of ovalbumin clearly favours lipid digestion by improving lipolysis and, in consequence, the bioaccessibility of some lipophilic nutrients. These outcomes confirm those results observed in previous works concerning the effect of the same ovalbumin proportion here tested on the lipolysis degree of slightly oxidized sunflower and flaxseed oils. ${ }^{4,6,7}$

Another noteworthy fact about the samples digested with a high proportion of ovalbumin is the important surge in the molar percentage of 2-MG in comparison with the rest of the digested samples. This reveals that, despite the high extent of TG hydrolysis observed in these samples, the increased lipolytic process does not seem to go beyond 2-MG; consequently, a great increase in glycerol molar percentage is not observed (see Table 3). The buffer capacity of ovalbumin might be involved in this finding, since it has been reported that a more alkaline $\mathrm{pH}$ in aqueous media can restrict the isomerization reactions of $2-\mathrm{MG}$ to $1-\mathrm{MG}$; $^{34}$ this would limit the MG hydrolysis to give rise to glycerol and fatty acids, since pancreatic lipase acts specifically on 1-MG. ${ }^{35}$ In this sense, it is worth mentioning that a higher $\mathrm{pH}$ was found during the gastric stage of the in vitro digestion process of the samples with a high proportion of ovalbumin in comparison with the rest of the samples. This potential influence of $\mathrm{pH}$, coupled with the higher hydrolysis of TG, and by extension, of 1,2-DG, might 
explain the accumulation of 2-MG; however, the influence of other factors should not be discarded either. This high proportion of 2-MG could in turn also contribute towards favouring the lipolytic process to a certain extent since, according to some authors, ${ }^{36}$ monoglycerides are highly surface-active products that can help to decrease the mean droplet diameter.

With respect to the molar percentages of 1,2-DG and 1,3DG, these are somewhat higher and lower, respectively, than in the samples digested without ovalbumin and with a small proportion of this protein. These outcomes might be a consequence of the enhanced lipolysis degree reached when digestion is performed in presence of a high ovalbumin level.

\subsection{Lipid bioaccessibility}

Of the parameters employed to evaluate the extent of lipolysis, lipid bioaccessibility $\left(\mathrm{L}_{\mathrm{BA}}\right)$ can be considered of particular interest, since it indicates the proportion of absorbable molecules (fatty acids and MG) with respect to the sum of acyl groups + fatty acids. ${ }^{3}$ While the addition of a low proportion of ovalbumin does not affect this parameter (see Table 3), when a high level of ovalbumin is present in the medium, $\mathrm{L}_{\mathrm{BA}}$ increases considerably, reaching values of $81.50 \%$ and $77.79 \%$ in the digestates of the virgin and of the refined oil samples, respectively. This indicates that the presence of this high proportion of protein can improve the absorption of dietary lipids, mainly due to the greatly increased 2-MG molar percentage.

\subsection{Assessment by ${ }^{1} \mathrm{H}$ NMR of lipid oxidation during in vitro digestion}

3.4.1. Study of the changes undergone by polyunsaturated acyl groups + fatty acids throughout in vitro digestion. The molar concentrations of the polyunsaturated groups in relation to the total of acyl groups + fatty acids in all the digested samples are shown in Table 1, together with those corresponding to the starting oils. Comparing the data before and after the in vitro digestion process, both in the absence and in presence of the two different ovalbumin proportions, statistically significant changes are not noticed. This reveals that, despite the initial oxidative degradation of the soybean oils here studied, oxidation occurrence during in vitro digestion cannot be inferred from acyl group degradation. However, this does not mean that some oxidation, undetectable through variations in the concentrations of polyunsaturated groups determined from ${ }^{1} \mathrm{H}$ NMR spectral data, has not taken place.

3.4.2. Study of the changes in the oxidation product profile caused by in vitro digestion

Evolution of hydroperoxide concentration. The in vitro digestion process causes a diminution in the concentration of hydroperoxides initially present in the oil samples, monitored through their associated conjugated dienes, this being more pronounced for the hydroperoxy- $(E, E)$-isomers than for the $(Z, E)$-ones (see Fig. 1 and Table 2). This does not necessarily mean that hydroperoxides are not generated during in vitro digestion, but that the rate of transformation overtakes that of formation. The same is observed when the samples are digested in presence of ovalbumin. However, while a low proportion of this protein does not significantly affect the hydroperoxide levels found after digestion, a more pronounced decline in the concentration of conjugated $(Z, E)$-, and especially of $(E, E)$-hydroperoxy-dienes occurs in the samples digested with a high ovalbumin proportion in comparison with the rest of digested samples.

At this point, it is worth noticing that conflicting results can be found about the stability and evolution of hydroperoxides in the gastrointestinal tract, as discussed by Márquez-Ruiz and coworkers. ${ }^{24}$ Thus, according to some authors, ${ }^{37,38}$ hydroperoxides decompose in the stomach giving rise to other oxidation products, while other researchers have proved that hydroperoxides can reach the intestine and then be absorbed. ${ }^{39}$ In our case, although hydroperoxide concentration diminishes during digestion, some part of them remains in the digestates.

Formation of conjugated hydroxy-dienes. The generation of both conjugated $(Z, E)$ - and $(E, E)$-hydroxy-dienes during digestion is observed in all the studied samples (see Fig. 1, signals "a" and "d", and Table 2). This suggests that a small part of the hydroperoxides present in the starting oils is reduced to hydroxy-dienes during digestion. Actually, the reduction of hydroperoxides to hydroxides throughout this process has also been reported previously both in vitro ${ }^{6}$ and in vivo. ${ }^{37-39}$ While an increase of a similar order is observed in the concentration of conjugated hydroxy-dienes in the digestates of oils without or with a low proportion of ovalbumin, this increase is noticeably greater in the digestates of the oils with a high ovalbumin level. This confirms previous findings ${ }^{4}$ and evidences the reducing effect of this protein.

Generation of epoxides. The concentration of epoxides exhibits an increment after digestion in all the studied samples (see Table 2), of a similar order in both types of soybean oils. This is due to an increase in the levels of the epoxides initially present in the starting oils, but also to the formation of other types of compounds, also tentatively identified as epoxides, which give signal "f" (see Fig. 1). Therefore, although hydroperoxides and aldehydes are generally employed to assess the occurrence of lipid oxidation under diverse conditions including gastrointestinal ones, ${ }^{8,9}$ epoxides should not be ignored as oxidation markers; this reinforces previous findings of this research group, since the generation of epoxides under in vitro digestion conditions had also been observed in slightly oxidized flaxseed oil. ${ }^{7}$

In line with all the above mentioned, the generation of epoxides during digestion does not seem to be influenced by a low ovalbumin concentration. However, a less pronounced increment of the level of epoxides is noticed when a high proportion of ovalbumin is present in the system (see Table 2).

Generation of aldehydes. As could be expected, taking into account that a depletion in hydroperoxide concentration is observed in the digested samples, aldehydes are generated during digestion. This is evidenced by the appearance of signals of $n$-alkanals in the spectra of all the digested samples (see signal "g" in Fig. 1); their estimated concentrations are 


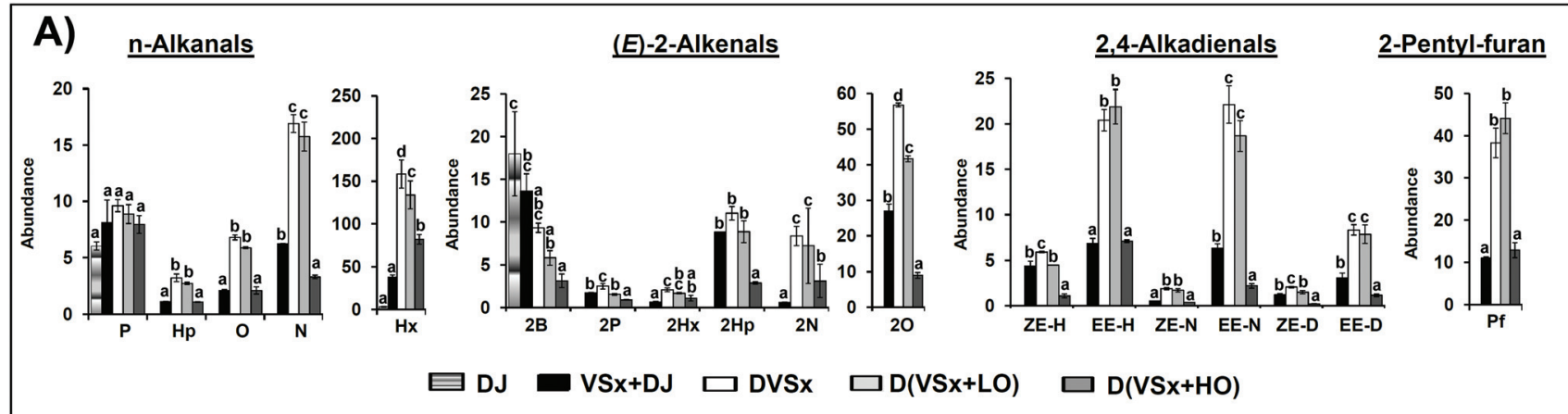

\section{B) n-Alkanals}

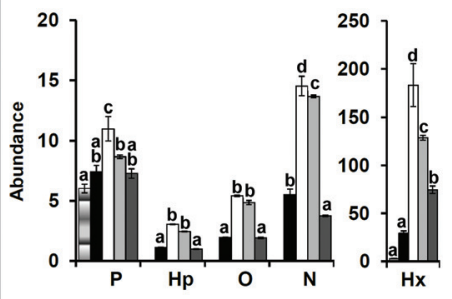

(E)-2-Alkenals

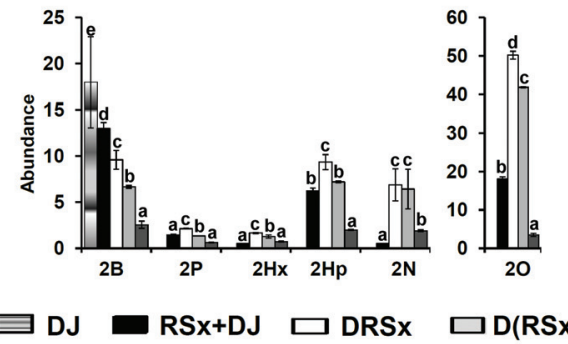

2,4-Alkadienals

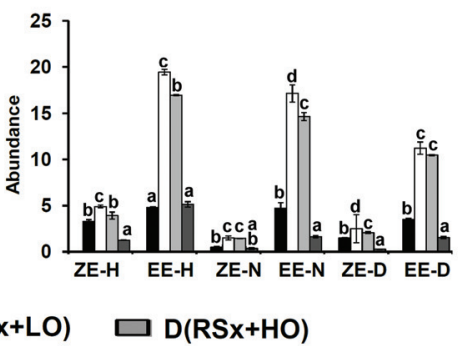

2-Pentyl-furan

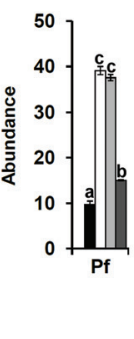

Fig. 2 Bar graphics representing the abundance, expressed as arbitrary area units of the mass spectrum base peak of each compound (see section 2.5.2) extracted from the total ion chromatograms obtained by SPME/GC-MS, divided by $10^{6}$, of $n$-alkanals, (E)-2-alkenals, 2,4-alkadienals and 2-pentyl-furan in: the digestive juices subjected to digestion conditions (DJ), the slightly oxidized virgin (A) and refined (B) oils mixed with the DJ (VSx + DJ and RSx + DJ), the slightly oxidized virgin and refined oils digested in the absence of ovalbumin (DVSx and DRSx), with a low proportion of ovalbumin (D(VSx $+L O)$ and $D(R S x+L O))$ and with a high proportion of ovalbumin $(D(V S x+H O)$ and $D(R S x+H O))$. Different letters within the bars relative to the same compound indicate a significant difference among the samples $(p<0.05)$. Abbreviations employed: pentanal $(\mathrm{P})$, hexanal $(\mathrm{Hx})$, heptanal $(\mathrm{Hp})$, octanal $(\mathrm{O})$, nonanal $(\mathrm{N}),(E)$-2-butenal (2B), (E)-2-pentenal (2P), (E)-2-hexenal (2Hx), (E)-2-heptenal (2Hp), (E)-2-octenal (2O), $(E)-2$ nonenal $(2 N),(Z, E)-2,4$-heptadienal $(Z E-H),(E, E)-2,4$-heptadienal $(E E-H),(Z, E)-2,4$-nonadienal $(Z E-N),(E, E)-2,4-$ nonadienal $(E E-N),(Z, E)-2,4-$ decadienal (ZE-D), (E,E)-2,4-decadienal (EE-D) and 2-pentyl-furan (Pf).

displayed in Table 2. The generation of $n$-alkanals during the in vitro digestion of slightly oxidized flaxseed oil was also reported in a previous study. ${ }^{7}$ Although the concentration of aldehydes found after digestion is not significantly affected by the low ovalbumin proportion tested, similarly to that commented on epoxides, lower levels of saturated aldehydes are observed after the digestion of the samples containing a high ovalbumin proportion. It is worthwhile mentioning that, as Table 2 shows, in all cases aldehydes are present in lower concentrations than epoxides.

In summary, the in vitro digestion of the slightly oxidized soybean oils and of these oils with a low ovalbumin level provokes very similar changes in the oxidation product profile of the starting oils. By contrast, when this protein is present in a high concentration, the reduction of hydroperoxides to hydroxy-dienes seems to be favoured over other reactions leading to the generation of epoxides and aldehydes. However, the lower concentrations of epoxides and saturated aldehydes in the samples digested with a high ovalbumin proportion in comparison with the rest of digested samples might also be due to their reaction with some amino acid residues of ovalbumin..$^{27,40}$

\subsection{Assessment of lipid oxidation through in vitro digestion by SPME-GC/MS}

The SPME-GC/MS analysis of the samples provides information about specific volatile compounds that can reveal the occurrence of different types of reactions, including oxidation. Among the several classes of volatile compounds present in the studied oils, interest was focused on aldehydes and 2-pentyl-furan. The compounds detected in the various digested samples and their respective abundances, expressed as arbitrary area units of the mass spectra base peak (see section 2.5.2), are displayed in Fig. 2, together with the data related to the reference samples prepared from the starting oils (see section 2.5.1), and to the digestive juices after being submitted to digestion conditions. The total abundances of each group of aldehydes are shown in Table 4.

As Fig. 2 shows, the reference samples already contain aldehydes, both saturated ( $n$-alkanals) and unsaturated $((E)$-2-alkenals and 2,4-alkadienals). Among $n$-alkanals, hexanal (Hx) stands out both in the virgin and in the refined oils due to its high level (Fig. 2A and B, respectively), probably as a consequence of some linoleic group oxidation. ${ }^{41}$ Regarding $(E)-2$ - 
Table 4 Total abundances of $n$-alkanals, (E)-2-alkenals and 2,4-alkadienals in: the digestive juices subjected to digestion conditions (DJ), the slightly oxidized virgin and refined oils mixed with DJ (VSX + DJ and RSx + DJ), and the slightly oxidized virgin and refined oils digested in the absence of ovalbumin (DVSx and DRSx), with a low proportion of ovalbumin added $(D(V S x+L O)$ and $D(R S x+L O))$ and with a high proportion of ovalbumin ( $(V S x+H O)$ and $D(R S x+H O))$. Different letters within each column indicate a significant difference among the samples corresponding to the same type of oil (virgin or refined) $(p<0.05)$

\begin{tabular}{lcll}
\hline & $n$-Alkanals & $(E)$-2-Alkenals & 2,4-Alkadienals \\
\hline DJ & $9.1 \pm 0.4 \mathrm{a}$ & $18.0 \pm 4.9 \mathrm{a}$ & - \\
& & & \\
VSx + DJ & $55.4 \pm 4.2 \mathrm{~b}$ & $52.3 \pm 2.2 \mathrm{~b}$ & $22.3 \pm 2.3 \mathrm{~b}$ \\
DVSx & $195.0 \pm 18.9 \mathrm{~d}$ & $90.1 \pm 3.7 \mathrm{c}$ & $60.7 \pm 4.0 \mathrm{c}$ \\
D(VSx + LO) & $167.1 \pm 20.8 \mathrm{~d}$ & $66.7 \pm 7.3 \mathrm{~b}$ & $56.1 \pm 5.0 \mathrm{c}$ \\
D(VSx + HO) & $96.2 \pm 6.8 \mathrm{c}$ & $20.1 \pm 3.5 \mathrm{a}$ & $12.1 \pm 0.8 \mathrm{a}$ \\
& & & \\
RSx + DJ & $45.4 \pm 2.3 \mathrm{~b}$ & $39.7 \pm 5.1 \mathrm{~b}$ & $18.3 \pm 1.1 \mathrm{~b}$ \\
DRSx & $217.0 \pm 22.3 \mathrm{e}$ & $79.8 \pm 3.7 \mathrm{~d}$ & $56.7 \pm 3.8 \mathrm{~d}$ \\
D(RSx + LO) & $158.2 \pm 2.5 \mathrm{~d}$ & $64.7 \pm 2.7 \mathrm{c}$ & $49.5 \pm 1.1 \mathrm{c}$ \\
D(RSx + HO) & $88.5 \pm 3.8 \mathrm{c}$ & $11.3 \pm 0.8 \mathrm{a}$ & $10.2 \pm 0.6 \mathrm{a}$ \\
-: not detected. & & & \\
& & & \\
\hline
\end{tabular}

alkenals and 2,4-alkadienals, the most abundant ones (apart from (E)-2-butenal, 2B, coming basically from the digestive juices) are (E)-2-octenal (2O), (E)-2-heptenal (2Hp), (E,E)-2,4nonadienal (EE-N) and (E,E)-2,4-decadienal (EE-D), mainly arising from the degradation of linoleic groups, as well as 2,4heptadienals (ZE-H and EE-H), generated due to linolenic acyl group oxidation. ${ }^{41}$ In addition to aldehydes, 2-pentyl-furan was also present in the samples studied.

When it comes to the digested samples, it can be observed in Fig. 2 that both in the samples digested without ovalbumin and with a low proportion of this protein, concentration of most $n$-alkanals increases significantly after in vitro digestion. However, for hexanal and nonanal (in this latter case only in the refined oil) the increase is less pronounced in the samples with a low ovalbumin level. By contrast, when a high ovalbumin proportion is present in the system, hexanal is the only $n$-alkanal that exhibits a concentration increase after digestion, while the rest either remain unchanged or show a decrease in relation to the non-digested samples (see Fig. 2). In fact, the total concentration of saturated aldehydes in the samples digested with a high ovalbumin proportion is lower than in the rest of digested samples (see Table 4). These findings follow the lines of those above obtained by ${ }^{1} \mathrm{H}$ NMR.

With regard to unsaturated aldehydes, the concentration of most (E)-2-alkenals and of all 2,4-alkadienals also increases after digestion in the samples digested without ovalbumin (see Fig. 2), although the global concentration increment of each group of unsaturated aldehydes is not as marked as in the case of the saturated ones (see Table 4). It must be highlighted that although ${ }^{1} \mathrm{H}$ NMR analysis does not allow one to notice the generation of unsaturated aldehydes, it is evidenced by SPME-GC/MS; this might be explained by their lower abundance in comparison with that of $n$-alkanals in the digested samples (see Table 4).
The same trend is observed in the samples digested with a low proportion of ovalbumin, where, as in the case of some $n$-alkanals, the overall increases of both types of unsaturated aldehydes are not so generally marked as in the samples digested without ovalbumin (see Table 4). However, in contrast to these findings, in the samples digested with a high ovalbumin proportion, the concentrations of $(E)$-2-alkenals and 2,4-alkadienals generally diminishes after digestion (see Fig. 2 and Table 4); this reinforces the idea noted above that reactions between aldehydes and ovalbumin take place during digestion. In this respect, the reaction of (E)-2-alkenals with proteins is a well documented issue. ${ }^{42}$ A reduction in aldehyde concentration during the in vitro digestion of other types of slightly oxidized highly unsaturated oils in presence of a high protein proportion was also observed in a previous work, ${ }^{4}$ but in that case data concerning the non-digested samples were not reported, so a direct comparison between these and the samples digested in presence of protein could not be made.

Comparing the effect of the high proportion of ovalbumin on the concentration of the various classes of aldehydes after digestion, and taking as a reference their respective levels in the samples digested without ovalbumin, it is noticeable that, in general, a more pronounced decrease is observed for the unsaturated aldehydes (see Table 4). This seems to agree with the greater reactivity that could be expected from this type of aldehydes in comparison with the saturated ones. Thus, the level of each aldehyde after the digestion process will depend on the balance between its generation and its ability to react with the proteins present in the medium.

In line with observations made for aldehyde evolution, the concentration of 2-pentyl-furan also increases after digestion, to a similar extent in the samples digested without ovalbumin and with a low ovalbumin proportion (see Fig. 2). However, in agreement with previous findings, ${ }^{4}$ the level of this compound is considerably lower in the samples digested with a high proportion of ovalbumin. Taking into account that, as far as we know, the ability of 2-pentyl-furan to react with proteins has not been described, this might be interpreted as that the presence of a high proportion of ovalbumin has exerted a certain antioxidant effect during digestion.

\subsection{Evolution of $\gamma$-T during the in vitro digestion process monitored by ${ }^{1} \mathrm{H}$ NMR}

The main tocopherol in soybean oil is $\gamma$-T. ${ }^{14}$ However, in these oxidized oils its presence could only be detected by ${ }^{1} \mathrm{H}$ NMR in the refined one (see signal "h" in Fig. 1, due to one of the protons present in the $\gamma$-T chromanol ring), its concentration being low: $0.63 \pm 0.06 \mathrm{mmol} \mathrm{mol}{ }^{-1} \mathrm{AG}+\mathrm{FA}$. After digestion, $\gamma$-T is only detected in the ${ }^{1} \mathrm{H}$ NMR spectrum of the sample digested with a high proportion of ovalbumin, although in a very low concentration $\left(0.07 \pm 0.01 \mathrm{mmol} \mathrm{mol}^{-1} \mathrm{AG}+\mathrm{FA}\right)$. This finding indicates, on the one hand, that, in agreement with previous results, ${ }^{15}$ the bioaccessibility of $\gamma$-T diminishes during in vitro digestion, and on the other that the presence of a high proportion of ovalbumin in the system preserves the $\gamma$-T 
content of the starting oil to a certain extent, possibly due to a decrease in oxidative reactions.

\subsection{Final remarks}

In order to make an approximate assessment of the overall oxidation extent during the in vitro digestion of the two types of slightly oxidized soybean oils studied, the total amounts of oxidation products considered to be supported on long acyl group or fatty acid chains (hydroperoxides, hydroxy-dienes and epoxides), were compared before and after this process. According to these data, increments in the total concentration of the considered oxidation products of about 1 and $2 \mathrm{mmol} \mathrm{mol}^{-1} \mathrm{AG}+$ FA in the case of the virgin and the refined oil samples, respectively, were observed after digestion. This suggests that, despite significant variations in acyl groups not being noticed after digestion (see Table 1), some oxidation seems to have taken place during this process.

Although a clear development of oxidative reactions was noticed during the in vitro digestion of slightly oxidized flaxseed oil in a previous work, ${ }^{7}$ it must be taken into account that it is more prone to oxidation than soybean oil simply due to its composition in acyl groups, ${ }^{43}$ although their composition in minor components might also have some influence. However, the results found after the in vitro digestion of slightly oxidized sunflower oil $^{6}$ are not very different from those here observed.

It is worth noticing that the small differences in the minor component profile of the slightly oxidized virgin and refined soybean oils relative to tocopherol, squalene and free fatty acid contents (see Table $\mathrm{S} 2 \dagger$ ) do not seem to be enough to provoke noticeable variations in their behaviour under in vitro digestion conditions.

\section{Conclusions}

The results of this work reveal that the initial oxidation degree of the soybean oil samples studied leads to a lower lipolysis extent in comparison with non-oxidized oils studied before, reducing the bioaccessibility of the oil major components, which include some essential fatty acids like $\omega-3$ ones. This lipolysis degree is not significantly affected by the lowest proportion of ovalbumin tested; however, the addition of a high ovalbumin level greatly enhances the action of lipolytic enzymes, improving lipid bioaccessibility.

Regarding oxidation, this does not seem to have occurred to a great enough extent for it to be detected from polyunsaturated acyl group degradation. Moreover, although the slight increase in the total concentration of oxidation products considered to be supported on acyl group or fatty acid chains after digestion might indicate some oxidation occurrence, the changes observed in the oxidation product profile of the digested samples seem to be mainly due to the transformation of the hydroperoxides initially present in the oils, to give hydroxy-dienes, epoxides and aldehydes. The fact that after digestion epoxides exhibit a greater concentration increase than aldehydes evidences that the assessment of lipid oxidation should not be based on only one group of oxidation products, but would require the determination of as many types of oxidation compounds as possible, among which epoxides should be included. While the presence of a low ovalbumin proportion during digestion does not significantly affect oxidation compound evolution, a high level of this protein favours the reduction of hydroperoxides to hydroxydienes over other type of reactions leading to the generation of epoxides and aldehydes. Notwithstanding, the decrease observed in the concentrations of hydroperoxides, epoxides and aldehydes might also be influenced by their reaction with the protein, and indeed, it has been evidenced by SPME-GC/ MS in the case of aldehydes.

Therefore, it is evident that a high proportion of ovalbumin, and possibly of other proteins, can play a key role in the digestion of lipids, especially in those showing a slight oxidation degree, increasing their absorbable fraction. In addition, the presence of a high proportion of ovalbumin not only increases $\gamma$-T bioaccessibility, but also contributes to reducing the concentration in the gastrointestinal tract of certain types of oxidation products that could exert negative effects, such as aldehydes and epoxides.

\section{Conflicts of interest}

The authors declare no conflict of interest.

\section{Acknowledgements}

This work has been supported by the Spanish Ministry of Economy and Competitiveness (MINECO AGL 2015-65450-R), by the Basque Government (EJ-GV, IT-916-16) and by the Agriculture Department of the Basque Government (PA19/ 02). A. S. Martin-Rubio thanks the MINECO for a predoctoral contract.

\section{References}

1 G. Fave, T. C. Coste and M. Armand, Physicochemical properties of lipids: new strategies to manage fatty acid bioavailability, Cell. Mol. Biol., 2004, 50, 815-832.

2 J. Kanner and T. Lapidot, The stomach as a bioreactor: Dietary lipid peroxidation in the gastric fluid and the effects of plant-derived antioxidants, Free Radicals Biol. Med., 2001, 31, 1388-1395.

3 H. B. Kenmogne-Domguia, A. Meynier, C. Boulanger and C. Genot, Lipid oxidation in food emulsions under gastrointestinal-simulated conditions: The key role of endogenous tocopherols and initiator, Food Dig., 2012, 3, 46-52.

4 B. Nieva-Echevarría, E. Goicoechea and M. D. Guillén, Effect of the presence of protein on lipolysis and lipid oxidation occurring during in vitro digestion of highly unsaturated oils, Food Chem., 2017, 235, 21-33. 
5 K. Larsson, L. Cavonius, M. Alminger and I. Undeland, Oxidation of cod liver oil during gastrointestinal in vitro digestion, J. Agric. Food Chem., 2012, 60, 7556-7564.

6 B. Nieva-Echevarría, E. Goicoechea, M. J. Manzanos and M. D. Guillén, ${ }^{1} \mathrm{H}$ NMR and SPME-GC/MS study of hydrolysis, oxidation and other reactions occurring during in vitro digestion of non-oxidized and oxidized sunflower oil. Formation of hydroxy-octadecadienoates, Food Res. Int., 2017, 91, 171-182.

7 B. Nieva-Echevarría, E. Goicoechea and M. D. Guillén, Behaviour of non-oxidized and oxidized flaxseed oils, as models of omega-3 rich lipids, during in vitro digestion. Occurrence of epoxidation reactions, Food Res. Int., 2017, 97, 104-115.

8 C. Steppeler, J. E. Haugen, R. Rødbotten and B. Kirkhus, Formation of malondialdehyde, 4-hydroxynonenal, and 4-hydroxyhexenal during in vitro digestion of cooked beef, pork, chicken, and salmon, J. Agric. Food Chem., 2016, 64, 487-496.

9 H. B. Kenmogne-Domguia, S. Moisan, M. Viau, C. Genot and A. Meynier, The initial characteristics of marine oil emulsions and the composition of the media inflect lipid oxidation during in vitro gastrointestinal digestion, Food Chem., 2014, 152, 146-154.

10 M. D. Guillén and E. Goicoechea, Toxic oxygenated $\alpha, \beta$-unsaturated aldehydes and their study in foods: a review, Crit. Rev. Food Sci. Nutr., 2008, 48, 119-136.

11 R. S. Lam and M. T. Nickerson, Food proteins: a review on their emulsifying properties using a structure-function approach, Food Chem., 2013, 141, 975-984.

12 R. J. Elias, S. S. Kellerby and E. A. Decker, Antioxidant activity of proteins and peptides, Crit. Rev. Food Sci. Nutr., 2008, 48, 430-441.

13 T. Van Hecke, J. Vanden Bussche, L. Vanhaecke, E. Vossen, J. Van Camp and S. De Smet, Nitrite curing of chicken, pork, and beef inhibits oxidation but does not affect $\mathrm{N}$-nitroso compound (NOC)-specific DNA adduct formation during in vitro digestion, J. Agric. Food Chem., 2014, 62, 1980-1988.

14 J. Alberdi-Cedeño, M. L. Ibargoitia, G. Cristillo, P. Sopelana and M. D. Guillén, A new methodology capable of characterizing most volatile and less volatile minor edible oils components in a single chromatographic run without solvents or reagents. Detection of new components, Food Chem., 2017, 221, 1135-1144.

15 A. S. Martin-Rubio, P. Sopelana and M. D. Guillén, Influence of minor components on lipid bioaccessibility and oxidation during in vitro digestion of soybean oil, J. Sci. Food Agric., 2019, 99, 4793-4800.

16 E. Goicoechea and M. D. Guillén, Analysis of hydroperoxides, aldehydes and epoxides by ${ }^{1} \mathrm{H}$ nuclear magnetic resonance in sunflower oil oxidized at 70 and $100{ }^{\circ} \mathrm{C}, \mathrm{J}$. Agric. Food Chem., 2010, 58, 6234-6245.

17 M. D. Guillén and A. Ruiz, Oxidation process of oils with high content of linoleic acyl groups and formation of toxic hydroperoxy- and hydroxyalkenals. A study by ${ }^{1} \mathrm{H}$ nuclear magnetic resonance, J. Sci. Food Agric., 2005, 85, 24132420 .

18 C. H. Versantvoort, A. G. Oomen, E. Van de Kamp, C. J. Rompelberg and A. J. Sips, Applicability of an in vitro digestion model in assessing the bioaccessibility of mycotoxins from food, Food Chem. Toxicol., 2005, 43, 31-40.

19 B. Nieva-Echevarría, E. Goicoechea, M. J. Manzanos and M. D. Guillén, A study by ${ }^{1} \mathrm{H}$ NMR on the influence of some factors affecting lipid in vitro digestion, Food Chem., 2016, 211, 17-26.

20 B. Nieva-Echevarría, E. Goicoechea, M. J. Manzanos and M. D. Guillén, Usefulness of ${ }^{1} \mathrm{H}$ NMR in assessing the extent of lipid digestion, Food Chem., 2015, 179, 182-190.

21 M. D. Guillén and A. Ruiz, Rapid simultaneous determination by proton NMR of unsaturation and composition of acyl groups, Eur. J. Lipid Sci. Technol., 2003, 105, 688-696.

22 M. D. Guillén and P. S. Uriarte, Contribution to further understanding of the evolution of sunflower oil submitted to frying temperature in a domestic fryer: study by ${ }^{1} \mathrm{H}$ Nuclear Magnetic Resonance, J. Agric. Food Chem., 2009, 57, 7790-7799.

23 B. Nieva-Echevarría, E. Goicoechea, M. J. Manzanos and M. D. Guillén, A method based on ${ }^{1} \mathrm{H}$ NMR spectral data useful to evaluate the hydrolysis level in complex lipid mixtures, Food Res. Int., 2014, 66, 379-387.

24 G. Márquez-Ruiz, M. C. García-Martínez and F. Holgado, Changes and effects of dietary oxidized lipids in the gastrointestinal tract, Lipid Insights, 2008, 2, 11-19.

25 F. J. Sánchez-Muniz, R. Arroyo, J. M. Sánchez-Montero and C. Cuesta, In vitro digestibility study of thermally oxidised palm oleins, Food Sci. Technol. Int., 2000, 6, 449-456.

26 H. W. Gardner, Lipid hydroperoxide reactivity with proteins and amino acids: a review, J. Agric. Food Chem., 1979, 27, 220-229.

27 K. M. Schaich, Co-oxidation of proteins by oxidizing lipids, Lipid Oxid. Pathways, 2008, 2, 181-272.

28 S. Matsushita and M. Kobayashi, Effect of linoleic acid hydroperoxides on pepsin activity, Agric. Biol. Chem., 1970, 34, 825-829.

29 S. Matsushita, M. Kobayashi and Y. Nitta, Inactivation of enzymes by linoleic acid hydroperoxides and linoleic acid, Agric. Biol. Chem., 1970, 34, 817-824.

30 S. Matsushita, Specific interactions of linoleic acid hydroperoxides and their secondary degraded products with enzyme proteins, J. Agric. Food Chem., 1975, 23, 150154.

31 G. Márquez-Ruiz, G. Guevel and M. C. Dobarganes, Applications of chromatographic techniques to evaluate enzymatic hydrolysis of oxidized and polymeric triglycerides by pancreatic lipase in vitro, J. Am. Oil Chem. Soc., 1998, 75, 119-126.

32 Y. Mine, T. Noutomi and N. Haga, Emulsifying and structural properties of ovalbumin, J. Agric. Food Chem., 1991, 39, 443-446.

33 C. Chen, Y. J. Chi, M. Y. Zhao and W. Xu, Influence of degree of hydrolysis on functional properties, antioxidant 
and ACE inhibitory activities of egg white protein hydrolysate, Food Sci. Biotechnol., 2012, 21, 27-34.

34 F. H. Mattson and R. A. Volpenhein, Synthesis and properties of glycerides, J. Lipid Res., 1962, 3, 281296.

35 F. H. Mattson and R. A. Volpenhein, The digestion and absorption of triglycerides, J. Biol. Chem., 1964, 239, 27722777.

36 A. M. Nik, A. J. Wright and M. Corredig, Interfacial design of protein-stabilized emulsions for optimal delivery of nutrients, Food Funct., 2010, 1, 141-148.

37 K. Kanazawa and H. Ashida, Catabolic fate of dietary trilinoleoylglycerol hydroperoxides in rat gastrointestines, Biochim. Biophys. Acta, 1998, 1393, 336-348.

38 K. Kanazawa and H. Ashida, Dietary hydroperoxides of linoleic acid decompose to aldehydes in stomach before being absorbed into the body, Biochim. Biophys. Acta, 1998, 1393, 349-361.
39 K. Nakatsugawa and T. Kaneda, Absorption and metabolism of methyl linoleate hydroperoxides in rats, J. Jpn. Oil Chem. Soc., 1983, 32, 361-366.

40 K. Ishino, T. Shibata, T. Ishii, Y. T. Liu, S. Toyokuni, X. Zhu, L. M. Sayre and K. Uchida, Protein N-acylation: $\mathrm{H}_{2} \mathrm{O}_{2}$ mediated covalent modification of protein by lipid peroxidation-derived saturated aldehydes, Chem. Res. Toxicol., 2008, 21, 1261-1270.

41 M. D. Guillén and P. S. Uriarte, Aldehydes contained in edible oils of a very different nature after prolonged heating at frying temperature: Presence of toxic oxygenated $\alpha, \beta$ unsaturated aldehydes, Food Chem., 2012, 131, 915-926.

42 K. Uchida, Aldehyde adducts generated during lipid peroxidation modification of proteins, Free Radical Res., 2015, 49, 896-904.

43 M. D. Guillén and A. Ruiz, Monitoring the oxidation of unsaturated oils and formation of oxygenated aldehydes by proton NMR, Eur. J. Lipid Sci. Technol., 2005, 107, 36-47. 